\title{
Review
}

\section{New Research Progress in Mycoplasma pneumoniae Infection}

\author{
Fengtan Li \\ Department of Radiology, Tianjin Medical University General Hospital, Tianjin, China
}

\author{
Keywords \\ Mycoplasma pneumoniae; infection; treatment; \\ immune response

\section{Correspondence} \\ Fengtan Li, \\ E-mail: ftantj@126.com \\ DOI: 10.1515/ii-2017-0114
}

\begin{abstract}
As an important pathogen of respiratory tract infection, Mycoplasma pneumoniae (MP) may not only lead to primary atypical pneumonia but also cause systemic organ conditions. MP causes respiratory tract infection among school-age children and is also an important infectious agent for adult respiratory tract infection. Intensive studies on roles of $M P$ infection on the innate immune system significantly aid development of targeted therapy drugs. Epidemic studies on associated symptoms also help in clinical prevention and diagnosis and show importance to personalized treatment utilizing different drugs for different patients. This study summarizes the abovementioned three points based on MP studies in recent years.
\end{abstract}

Mycoplasma pneumoniae (MP) infection is clinically manifested by severe intractable cough; it was formerly called primary atypical pneumonia (PAP) or pneumonia with positive cold agglutination, wherein basic pathogenesis involves interstitial lung disease or capillary bronchitis ${ }^{[1]}$. In 1944, MP was first separated from phlegm expectorated by a patient with PAP. MP infection may lead to lower respiratory tract infection (LRTI) in humans. $M P$ was mistaken for a virus until it was effectively killed with antibiotics in a recent study ${ }^{[2]}$. In 1961, Chanock successfully cultivated and classified MP in cell culture media ${ }^{[3]}$.

Mycoplasma orale is the most common host of symbiotic mycoplasmas in human oral-pharyngeal regions. Among symbiotic mycoplasmas in humans, $M P$ is the most carefully studied to date. Extensive investigations on $M P$ covered host immune responses, laboratory testing techniques, epidemiological studies, and pathogenesis of respiratory pathogens.

\section{Immune responses to $M P$}

$M P$ increases antibody $(\mathrm{Ab})$ responses of infected individuals through proteins and glycolipid antigens, with P1 protein serving as the main target of most host immune responses that induce $\mathrm{Ab}$ secretion; $\mathrm{MP}$ is also an important indicator for serological tests ${ }^{[4]}$. At the initial stage of $M P$ infection, normal immune system produces Abs six weeks after reaching the peak and phases down production of Abs in a few months or after several years ${ }^{[5]}$. MP infection often displays apparent symptoms when clinically producing antigen/antibody reactions ( $\mathrm{Ab}-\mathrm{Ag}$ reactions) because of long-term latency of the organism. Large quantities of $M P$-specific $\operatorname{IgM}$ are often interpreted as evidence of acute infection because these Abs are frequently observed within the first week of initial onset of infection and first two weeks of IgG-Ab production. Typically, children are first exposed to $M P$ infection, with IgMs appearing and changing the most significantly. Adults do not present IgG responses to $M P$ antigen within several years after infection ${ }^{[6]}$. IgA, which is often neglected as diagnostic $\mathrm{Ab}$, is actually an indicator of latest onset of $M P$ infection. $\operatorname{IgA}$ is produced early during the course of the disease and may rapidly increase to peak value and decrease slowly ahead of $\operatorname{IgM}$ and $\operatorname{IgG}{ }^{[7]}$. IgA level in adults and children may be detected in body fluids, including urine. An intact immune system plays an important role in preventing infection and transmission of $M P$ disease. $M P$-specific $\mathrm{Abs}$ and $\mathrm{Abs}$ involving cross reactions pose synergistic effects on MP. Membranes from mammalian tissues show sequence homology to $M P$ adhesin proteins and glycolipids. A variety of autoimmune diseases may be 
caused by molecular mimicry, and sites of diseases depend upon tissue structures, including myosin, horn, fibrinogen, brain, liver, kidney, smooth muscles, and lung tissues, that respond to $\mathrm{Abs}{ }^{[8]}$. MP adhesin and human CD4 cells and type II major histocompatibility complex lymphocyte protein also exhibit amino acid sequence homology, which may lead to production of autoreactive antibodies and trigger necrocytosis and immunosuppression ${ }^{[9]}$. Specific T cellmediated immunity also participates in host responses to $M P$ infection. After culturing in medium containing $M P$, lymphocytes from the human body were provided for blast transformation ${ }^{[10]}$. In the presence of microorganisms, white blood cells from $M P$-infected population exhibit chemotaxis. Most mycoplasma-infected animals or humans may induce chronic diseases. Fully understanding MP poses extremely difficulty. These prokaryotic microbes may successfully evade host immune responses by evolutionary processes. Thus, neither intracellular location nor immunoregulation may play roles in such case. Another mechanism is antigenic changes on other bacterial surfaces. In $M P$, surfaceantigen hypermutation of adhesin protein, DNA variation and recombination of $\mathrm{P} 1$ adhesin, and three $\mathrm{P} 1$ adhesin operons may further encode genomes of a limited number of mycoplasmas and boost their diversity and specificity ${ }^{[11]}$.

\section{Epidemiological studies and symptoms of MP infection}

Over $40 \%$ of community-acquired pneumonia (CAP) is caused by $M P$, and no less than $18 \%$ of child cases require hospitalization ${ }^{[12]}$. MP pneumonia (MPP) rarely occurs among children below five years old and mostly affects teenagers at the age of 5-15. Incidences of the disease among adolescent and adult patients gradually decrease with age. MPP may occasionally exist in some areas or among elderly and children under the age of five ${ }^{[13]}$. MP is detected in $23 \%$ of 3-4-year old children with CAP ${ }^{[14]}$. Although MP is usually not defined as a neonatal pathogen, PCR showed that $M P$ may transmit infectious nasopharyngeal secretions via the placenta and cause congenital MPP to newborns ${ }^{[15]}$.

Pneumonia is the most serious disease induced by $M P$ infection, with the most typical symptoms of tracheobronchitis, especially in child cases with various symptoms of upper respiratory infection tract (URTI). Studies showed that $23 \%$ of children with acute $M P$ infection also suffer from nonstreptococcal pharyngitis
[16]. MP infection is generally mild. Many adults exhibit no symptoms, which are less common in children. These findings reflect protective immunity against recurrence of infection to some extent. Normally, secondary infection is extremely mild to cause pneumonia. Although mycoplasma infections are mostly curable in outpatient clinics, $M P$ infection remains a significant cause of adult bacterial pneumonia, which requires hospitalization. Cystic fibrosis assay for antibody detection indicated that MP may cause hospitalization in $5.4 \%$ of patients with CAP. Incidence of $M P$-induced pneumonia among adults increases with age. However, among the elderly, MP is a pathogen that causes pneumonia and is second only to Streptococcus pneumoniae. Another study showed that MP accounts for $29.2 \%$ of all pathogens inducing pneumonia ${ }^{[17]}$.

MP infection may be manifested with URTI, LRTI, or both. Frequency of nonspecificity of URTI symptoms varies from literature to literature. Some reports indicated that $50 \%$ of patients with MP are infected with URT diseases ${ }^{[18]}$. Symptoms of $M P$ infection often manifest within several days after infection and last for a couple of weeks or months. The most common symptoms include odynophagia, hoarseness, fever, and cough. With disease progression, $M P$ infection may cause production of small or moderate amounts of bloody sputum, headache, chill, runny nose, myosalgia, earache, general malaise, and in very severe cases, difficulty in breathing, whooping cough, and chest pain, especially lasting pediatric cough that sometimes cause sore throat, adenopathy, conjunctivitis, and myringitis. Children under the age of five may exhibit nasitis and asthma, but they are generally not susceptible to pneumonia. However, those at the age of 5-15 may possibly suffer from bronchopneumonia and may sometimes require hospitalization. Mild infection and asymptomatic MP infection are commonly observed in adults ${ }^{[19]}$. As mentioned above, $M P$ is a leading cause of severe pneumonia requiring hospitalization, especially among the elderly and results in 5\% of bronchiolitis among children ${ }^{[20]}$. MPP with sporadically or locally sonorous rales and wheezing rales may be revealed by auscultation. In absence of complications, acute fever lasts for a week or so, and cough and lassitude may continue for two weeks or longer. When antibacterial therapy is provided at early stages of the disease, duration of $M P P$ symptoms is generally short.

Clinical manifestations of $M P$-induced respiratory diseases are often similar to those of other atypical infections caused by pathogens, especially Chlamydia pneumoniae 
and various respiratory viruses and bacteria, such as $S$. pneumoniae. Respiratory MP infection may also accompany other pathogens. MP infection in humans and mammals is followed by those of various respiratory viruses and bacteria, including Streptococcus and Neisseria meningitidis ${ }^{[21]}$.

\section{Treatment of MP}

MPP is a self-limited disease that may be automatically cured without requiring antibiotics. However, current studies suggested that antibiotics may ease symptoms, shorten the course of disease, and avoid occurrence of complications ${ }^{[22]}$. Mycoplasma is a genus of bacteria that are in the size between bacteria and viruses and is one of the smallest known pathogenic microbes that can live independently and pass through bacteria-retaining filters. Penicillin, cephalosporin, and other antibiotics acting on cell walls cannot affect $M P$ ${ }^{[23] . ~ M P ~ a r e ~ h i g h l y ~ s e n s i t i v e ~ t o ~ d r u g s, ~ i n c l u d i n g ~ m a c r o l i d e, ~}$ tetracycline (TC), and fluoroquinolones (FQNS). Among these drugs, TC and FQNS are restricted for use because of their serious side effects. Macrolide antibiotics contains a 14-16-membered large lactone ring as mother nucleus and are each linked with 1-3-molecule sugar using glycosidic bonds formed by hydroxyl radicals ${ }^{[24]}$. Macrolide antibiotics may kill mycoplasma by suppressing protein synthesis ${ }^{[25]}$. Bacterial protein is synthesized in ribosomes, whereas macrolide antibiotics may be combined with a special target of $23 \mathrm{~S}$ ribosomal RNA of the $50 \mathrm{~S}$ ribosomal subunit of bacteria and certain ribosomal proteins. Peptide extension is suppressed by mechanically blocking passages, thus hindering synthesis of protein. Macrolide antibiotics also inhibit assembly of the 50S ribosomal subunit, displaying a reduction in population of functional ribosomes, poor ability to synthesize mycoplasmas protein, and inhibited bacterial growth ${ }^{[26]}$.

Tissue injuries from $M P$ infection are inclusive of those caused by direct immunofluorescence and inflammatory mediators. Among these mediators, released inflammatory mediators contain acid hydrolases, natural proteolytic enzymes, and lysosomal enzymes and are involved in reduced humoral immunity and increased cellular immunity. Adrenocortical hormones may be used by patients with atelectasis, pulmonary interstitial fibrosis, bronchiectasia or extrapulmonary complications arising from rapid progression of the disease and at acute phase, severe $M P$ infection, or persistent pulmonary lesions. High-dose steroid treatment may effectively reverse nervous system symptoms of $M P$-infected children. Steroids combined with antibiotics, such as doxycycline or chloramphenicol, may effectively penetrate the central nervous system ${ }^{[27]}$. Azithromycin (AM) and clarithromycin are often used in treating MPinduced RTI. Although macrolide drugs are poorly assimilated into the central nervous system, they are still successfully used in treating MP-induced central nervous system infection. Among 14 cases with severe $M P$-induced central nervous system diseases, 11 cases (78\%) completely or nearly completely recovered, and these results were much better than those without combined steroids ${ }^{[28]}$.

Prophylactic antibiotics (PAs) are used to control epidemic situation of MP-infected diseases and function effectively at varying degrees ${ }^{[29]}$. TCs were used in some previous studies. New macrolide antibiotics were applied in current studies. AM was effectively used to prevent and treat $M P$ infection in clinical practice, with protective effect of $75 \%$ as revealed by hospital staff ${ }^{[30]}$. Serological tests showed that administrating $500 \mathrm{mg}$ AM per week may produce protective effects by on $64 \%$ of MP infection. Use of PAs may efficiently cope with $M P$ outbreak ${ }^{[31]}$. Table 1 lists minimal inhibitory concentration susceptibility data of commonly used antibiotics.

As a result of extensive use of antibiotics, drug-resistant strains are produced abundantly. Accordingly, traditional Chinese medicines become increasingly important in treating $M P$ infection. In traditional medicine, pneumonia, which belongs to warm diseases, may be treated by dispersing lung qi and relaxing bowels and clearing liver fire while considering the viewpoint of "the interiorexterior pattern relationship between the lung and the large intestine." The disease is caused by latent phlegm---heat in viscera interact with turbid qi in the large intestine because of exogenous pathogenic factors involving lungs and with exogenous heat invading the Upper Jiao, body fluid loss, and sputum accumulation; thus, treating pediatric $M P P$ by dispersing lung qi and relaxing bowels is recommended ${ }^{[32]}$. Removal of heat and toxins ventilates lungs and resolves phlegm problems ${ }^{[33]}$. According to pathogenesis of warm diseases, "toxins that lie in pathogenic factors and invade with pathogenic factors produce heat and cause diseases." Eliminating pathogenic factors and detoxifying the body play crucial roles in in treating pneumonia. Lungs are the primary organs involved in circulation of Qi blood, and once exogenous pathogenic factors invade these organs, they are 
inclined to Qi blood stagnation. Drugs that promote blood circulation and remove blood stasis may be objectively used for treatment. Combined use of drugs that eliminate heat and toxins and promote blood circulation to remove blood stasis may strengthen nonspecific antiinfection drugs.

Lack of protection of corresponding Abs in MP-infected body leads to large-scale prevalence and $M P$ outbreak. Thus, appropriate Abs against MP must be developed. After $M P$ was identified and isolated, $M P$ vaccine was developed in the 1970s. Waites et al. ${ }^{[34]}$ explored MP-related immune responses using animal models and mapped out preliminary vaccine design, under which formaldehydeinactivated $M P$ was injected into the body of volunteers for immunostimulation but without achieving ideal effects. To date, both mycoplasma vaccine and attenuated live vaccine are still at their respective experimental stages.

\section{Prospects}

In current studies, $M P$ and its related diseases were detected by PCR, serological tests, in vitro culture, and genomic sequencing; these research contents were applied to epidemiological investigations, animal models, evaluation of diagnostic reagents, and clinical trials of antimicrobial agents ${ }^{[35]}$. Considerable achievements were obtained over the past few decades, and these advances covered cellular biological characteristics of $M P$, reactions with host cells, pathogenesis of disease, escape mechanism from host defense system, spread of disease, treatment of chronic lung diseases, and development of new antimicrobial agents. However, various issues still require further studies. No quick, sensitive, specific, or reasonably priced method or means of direct detection can clinically treat MP infection. To date, serological test is the only method for diagnosing $M P$ infection, but it still features certain limitations. A proven and user-friendly detection method that measures mycoplasma content or nucleic acid in clinical specimens bears significance in diagnosis and management of patients and studies on MP in cases with chronic lung diseases and related antibiotics. However, further research should still focus on developing safe and protective vaccines that effectively reduce risk of MP infection among high-risk populations.

\section{Declarations}

\section{Acknowledgements}

No.

\section{Competing interests}

The author declares that he has no competing interest.

\section{Authors' contributions}

FT Li made the literature analysis and wrote, discussed and revised the manuscript of this review.

\section{References}

1 Park IH, Choi du Y, Oh YK, et al. A case of acute myopericarditis associated with Mycoplasma pneumoniae infection in a child. Korean Circ J, 2012, 42(10):709-13.

2 Abele-Horn, W.Franck, U.Busch, et al. Transverse myelitis associated with Mycoplasma pneumoniae infection. Clin Infect Dis, 1998, 26(11):909-12.

3 Kung CM, Wang RH, Wang HL. High prevalence of Mycoplasma pneumoniae in hepatitis $\mathrm{C}$ virus-infected hemodialysis patients. Clin Lab, 2012, 58(9):1037-43.

4 Arav-Boger R, Assia A, Spirer Z, et al. Cholestatic hepatitis as a main manifestation of Mycoplasma pneumoniae infection. Pediatr Gastroenterol Nutr, 1995, 21(4):459-60.

5 Li Changzhen, Rao Jingjing, Wang Rong et al. Analysis of Non-bacterial Pathogen Infection among Children with Wheezing Disorders Chinese Journal of Contemporary Pediatrics, 2012,14(11):834-7.

6 Aubert, G.Pozzetto, B.Hafid, et al. Immunoblotting patterns with Mycoplasma pneumoniae of serum specimens from infected and noninfected subjects. J Med Microbiol, 1992,36 (4):341-6.

7 Kong Min, Jiang Li, Hu Jun et al. Clinical Characteristics and Literature Review of Ischemic Stroke Induced by Pediatric MP Infection.Chinese Journal of Contemporary Pediatrics, 2012, 14(11):823-6.

8 Chen L, Cheng Y, Chen Z, et al. Studies on Coinfection of Mycoplasma Pneumoniae Pneumonia in Children. Chinese Journal of Pediatrics, 2012,50(3):211-5

9 Yuan Z, Dong Z, Lu J, et al. Several Problems in the Diagnosis and Treatment of Pediatric Mycoplasma Pneumoniae Pneumonia. Chinese Journal of Practical Pediatrics, 2002, 17(8):449-57.

10 Uldum SA, Amberg P, Jensen JS. Macrolide resistance in Mycoplasma pneumoniaeby recurrence and extended illness. Ugeskr Laeger, 2012, 174(11):730-41.

11 Yan X, Zhao F, Zhang J. Establishment and Application of New p1 Genotype Mycoplasma Pneumoniae Typing Method. Journal of Microbiology, 2012,52(2):262-7.

$12 \mathrm{X}$ in D, Ma H, Hou A, et al. Experimental Studies on the In Vitro Inhibitions of Different Heat/Toxin-Clearing Formulae on Mycoplasma 
Pneumoniae. Chinese Journal of Experimental Traditional Medical Formulae, 2004,10(5):43-5.

13 Youn YS, Lee KY. Mycoplasma pneumoniae pneumonia in children. Korean J Pediatr, 2012, 55(2):42-7.

$14 \mathrm{Xu} \mathrm{C}$, Zhao Z. Present Situation and Outlook of Treating Pediatric Mycoplasma Pneumonia with TCM. Shizhen China, 2006,17(12):2591-2.

15 Hong SJ. The Role of Mycoplasma pneumoniae infection in Asthma. Allergy Asthma Immunol Res, 2012, 4(2):59-61.

16 Dong Z. Treatment of Pediatric Mycoplasma Pneumoniae Pneumonia. Chinese Pediatric Emergency Medicine,2002,9(3):137-9.

17 Zhang B. Observations and Follow-up Visits of Pycoplasma Pneumoniae Pneumonia Treated with Different Drugs. Chinese Journal of Contemporary Pediatrics, 2001,3(6):695-6.

18 Yuan H, Zhang Q, Li Z, et al. Studies on Mycoplasma Pneumoniae and Chlamydia Infection in Children with Respiratory Tract Infection. Journal of Xinxiang Medical College, 2003, 20(4):254-5.

19 Koga S, Ishiwada N, Honda Y, et al. A case of meningoencephalitis associated with macrolide-resistant? Mycoplasma pneumoniae? infection.Pediatr Int, 2012, 54(5):724-6.

20 Huang $H$, Zhang Y, Huang S, et al. Etiological Investigations on Community-acquired Pneumonia. Chinese Journal of Infection and Chemotherapy, 2003, 3(6):321-4.

21 Trcko K, Marko PB, Miljkovic J. Leukocytoclastic vasculitis induced by Mycoplasma pneumoniae? infection. Acta Dermatovenerol Croat, 2012, 20(2):119-22.

22 Othman N, Isaacs D, Kesson A. Mycoplasma pneumoniainjection in Australia children.J Paediatr Child Health, 2005, 41(12):671-6.

23 Zhu Y, Xu H, Yue Z, et al. Detection Analysis on Mycoplasma Pneumoniae Abs in Patients with Respiratory Tract Infection. Journal of Pathogen Biology, 2011, 6(6):478-9.

24 Saegeman V, Proesmans M, Dumke R. Management of Macrolideresistant?Mycoplasma pneumoniaeInfection. Pediatr Infect Dis J, 2012,
31(11):1210-21.

25 Morozumi M, Ubukata K. Macrolide-resistant Mycoplasma pneumoniae. Nihon Rinsho, 2012,70(2):251-5.

26 Shen Hui, Shen Ce. Research Progress of the Immunoregulation of Macrolide Drugs. Section of Respiratory System: Foreign Medical Sciences, 2005,25(11):818-21.

27 Zhu Q, Xu J, Liu J. Epidemiological Studies on Pediatric Mycoplasma Pneumoniae in Qingdao. Chinese Journal of Nosocomiology, 2004,14(3):248-50.

28 Yu Zhiling, Yuan Zhuang \& Liu Chunhui.Clinical Analysis on 22 Cases with Central Nervous System Disorders Induced by Mycoplasma Pneumoniae Infection.Chinese Journal of Practical Pediatrics,2000,15(8):495-6.

29 Chen Q, Zhang Q. 1,834 Cases with Mycoplasma-infected Genitourinary Tracts and Drug Susceptibility Results Analysis. Journal of Pathogen Biology, 2013,8(1):173-4,183.

30 Yu Yan \& Li Jixiang.Comparision between Azithromycin and Erythromycin in Treating Mycoplasma Pneumonia.Chinese Journal of Nosocomiology,2004,14(5):570.

31 Tang H, Lin Y. Analysis of the Resistance of Mycoplasmas to Commonly Used Antibiotics. Chinese Journal of Nosocomiology, 2002, 12(8):578-9.

32 Shin SR, Park SH, Kim JH, et al. Clinical Characteristics of Patients with Mycoplasma pneumoniae-related acute hepatitis. Digestion, 2012, 86(4):302-8.

33 De Lalibera IB, Silveira Gde A, Toma RK, et al. Meningoencephalitis associated with Mycoplasma pneumoniae. Einstein(Sao Paulo), 2012,10(1):100-2.

34 Waites KB, Talkington DF. Mycoplasma pneumonia and its roleas a human pathogen. Clin Microbiol Rev, 2004, 17(4):697-728.

35 Ren Yi, Li J, Tian X, et al. New Progress of Understanding Mycoplasma Pneumoniae Infection. Journal of Pathogen Biology, 2012, $7(7): 550-1,492$. 